\title{
Probióticos como posible apoyo en el tratamiento de la periodontitis crónica
}

\section{Probiotics as a possible support in the treatment of chronic periodontitis}

\author{
Donald Ramos-Perfecto $^{1 *}$, Cinthia Berrocal-Medrano², Adelmo Cuentas-Robles ${ }^{1}$, Américo Castro-Luna $^{3}$
}

\author{
1 Docente de la UNMSM Facultad de Odontología, \\ Perú. \\ 2 Cirujano Dentista UNMSM, Perú. \\ 3 Docente de la UNMSM Facultad de Farmacia y \\ Bioquímica, Perú. \\ * Correspondencia Autor: Mg Donald Ramos P. \\ Facultad de Odontología Av. German Amezaga s $n$. \\ Lima 1. Perú. | e-mail: dramosp@unmsm.com.pe \\ Trabajo recibido el 25/06/2017. \\ Aprobado para su publicación el 10/09/2017
}

\begin{abstract}
RESUMEN
Se realizó una revisión bibliográfica cuyo objetivo es dar a conocer los beneficios de los probióticos en su posible uso en la periodontitis crónica. En la actualidad han aparecido nuevos enfoque terapéuticos, que podrían complementar el tratamiento de la periodontitis crónica. Es bien sabido que el gold estándar en el tratamiento no quirúrgico, es el raspado y alisado radicular y que en su apoyo pueden haber coadyuvantes, como lo podrían ser los probióticos. Estos son microorganismos comensales de la microflora bucal que siendo administrados de forma adecuada, brindarían beneficios a la persona, como la mejora de su salud periodontal. Los probióticos son productores de sustancias antimicrobianas, tienen un mecanismo competitivo con las bacterias patógenas, así como moduladores de la respuesta inmune. En esta revisión se detallan sus múltiples beneficios así como su apoyo al tratamiento de la periodontitis crónica.
\end{abstract}

PALABRAS CLAVE

Probióticos; Periodontitis crónica; Tratamiento.

Rev. Clin. Periodoncia Implantol. Rehabil. Oral Vol. 11(2); 112-115, 2018.

\section{ABSTRACT}

A bibliographic review was carried out to present the benefits of probiotics in their possible use in chronic periodontitis. At present, new therapeutic approaches have appeared, which could complement the treatment of chronic periodontitis. It is well known that the gold standard in non-surgical treatment is scaling and root planing and that adjuvants, such as probiotics may support it. These commensal microorganisms of the oral microflora , when administered properly, would provide benefits to the individual, such as improving their periodontal health. Probiotics are producers of antimicrobial substances and have a competitive mechanism with pathogenic bacteria, as well as being modulators of the immune response. This review details their multiple benefits and their support in the treatment of chronic periodontitis.

\section{KEY WORDS}

Probiotics; Chronic periodontitis; Treatment.

Rev. Clin. Periodoncia Implantol. Rehabil. Oral Vol. 11(2); 112-115, 2018.

\section{INTRODUCCIÓN}

Los probióticos son microorganismos vivos que administrados de forma adecuada confieren un beneficio de salud al huésped ${ }^{(1-3)}$. Siendo los más mencionados en la literatura los géneros Lactobacillus y Bifidobacterium. Estos son microorganismos comensales del huésped y que su sola presencia permite estabilizar la flora microbiana y modular una respuesta inmune positiva para el individuo ${ }^{(4)}$. Aunque un concepto más común en referencia a los probióticos es ser un preparado o un producto que contiene cepas de microorganismos viables en cantidades suficientes como para alterar la microflora en algún compartimento del huésped produciendo un beneficio(5,6). En la actualidad los probióticos son una opción para evitar el desbalance que se genera con el consumo de antibióticos, principalmente en el aumento de la resistencia bacteriana y alteraciones en el intestino grueso, que se evitarían con el uso de microorganismos comensales que brindarían una salud general y periodontal del huésped $\left.{ }^{(3,7,8}\right)$. Si bien es cierto que los antibióticos son una opción que complementa el tratamiento periodontal, ya que su administración sistémica reduce la profundidad de la bolsa periodontal en $0,3 \mathrm{~mm}$ y gana en $0,2 \mathrm{~mm}$ el nivel de inserción clínica, mientras que la aplicación local brinda un beneficio de reducción en 0,4 mm de la bolsa periodontal y una ganancia de $0,3 \mathrm{~mm}$ del nivel de inserción clínica ${ }^{(9)}$, otras opciones como el uso de los probióticos podrían ser de utilidad en el tratamiento. Resultados prometedores se han podido obtener al complementar la terapia periodontal con probióticos, principalmente al reducir las dimensiones de la bolsa periodontal, en pacientes con periodontitis crónica ${ }^{(7,10,11)}$.

Los probióticos no son antibióticos, si no, microorganismos que promueven bacterias comensales en el organismo e inhiben bacterias patógenas como la Porphyromonas gingivalis ( $P$. gingivalis) ${ }^{(12)}$ que tiene gran relevancia en la periodontitis crónica ${ }^{(13)}$.

\section{HISTORIA}

La palabra probiótico deriva del latín que significa a favor de la vida, que fue utilizado por Elie Metchnikof en 1907, quien propuso que la longevidad de los campesinos balcánicos estaba asociada al consumo de productos lácteos fermentado, siendo Lilly y Stilwell en 1965, quienes utilizaron la palabra para conceptualizar una sustancia secretada por microorganismos que estimulan el crecimiento de otro. Parker en 1974 
acuña el término probiótico, con el concepto de organismos y sustancias que contribuyen al equilibrio de la microbiota intestinal ${ }^{(1,2)}$.

\section{CARACTERÍSTICAS}

Los probióticos son microorganismos saprofitas o comensales, resistentes al pH gástrico y a las secreciones pancreáticas, con capacidad de adherirse a las células epiteliales así como inhibir la adhesión de microorganismos virulentos, con gran actividad antibacteriana para flora patógena, así como no poseer genes de resistencia a los antibióticos ${ }^{(1,2,10,14,15)}$.

\section{CLASIFICACIÓN DE LOS PROBIÓTICOS}

Los probióticos se pueden clasificar según ciertas características de los microorganismos, como la producción de una sustancia, ausencia o presencia de características no constantes en la bacteria. Principalmente perteneciente a los géneros bacterianos de Lactobacillus, Bifidobacterium, Streptococcus, Propionibacterium y algunas levaduras ${ }^{(1,14)}$.

a) Probióticos productores de sustancias ácido lácticas (Lactobacillus reuteri, Bifidobacterium bifidum)

b) Probióticos productores de sustancias no ácido lácticas ( Propionibacterium)

c) Levaduras no patógenas ( Saccharomyces boulardii)

d) Bacterias no formadoras de esporas (cocobacilos)

\section{Formas de presentación de los probióticos}

Se puede encontrar probióticos en presentaciones de bebidas (jugo de frutas), comida (queso), fibras prebióticas, productos lácteos, productos empaquetados con probióticos secos o deshidratados pero viables, en forma de polvo, cápsula, gelatina, tabletas, enjuagues bucales, etc ${ }^{(1,14)}$.

\section{Mecanismo de acción}

a) Los probióticos liberan componentes antimicrobianos como ácidos orgánicos, ácidos grasos libre, peróxido de hidrógeno y bacteriocinas, este último es un péptido catiónico sintetizado por los ribosomas, los cuales destruyen bacterias, así la bacteriocina de Lactobacillus paracasei HL32 es capaz de destruir $P$. gingivalis, formando poros a nivel de su cápsula ${ }^{(16)}$.También la bacteriocina de Lactobacillus plantarum NC8 y 44048 , inhiben $P$. gingivalis patógeno predominante de la periodontitis crónica al causar distorsión celular a través de la separación de la membrana externa y la lisis bacteriana ${ }^{(17)}$.

b) La producción de compuestos ácidos reducen el $\mathrm{pH}$ del medio ambiente, inhibiendo el crecimiento de organismos patógenos ${ }^{(18)}$.

c) Exclusión competitiva, concepto que se entiende como la competencia de recursos por dos especies diferente, que ante la competencia se va a dar la extinción de uno de ellos. Esto puede ocurrir impidiendo la adhesión de bacterias patógenas o compitiendo por los mismos nutrientes. Los Streptococcus impiden la colonización de patógenos periodontales a la superficie de tejidos duros y blandos, por la producción de sustancias biosurfactantes. Así también cepas probióticas inhiben la adhesión mediante la modificación de la composición de la proteína del sitio de unión(19).

d) Modulación del sistema inmune, los probióticos o sus productos actúan sobre una variedad de células para modular el sistema inmune y ejercer una acción antiinflamatoria. Se ha propuesto que los probióticos comensales activan células $\mathrm{T}$ reguladoras que inducen tolerancia por la activación de la interleuquina (IL)-10 y el factor de crecimiento transformante beta, además interaccionan selectivamente con receptores tipo Toll que regulan la liberación de citoquinas proinflamatorias, por lo tanto modulando la respuesta inmune. Así también inducen a los queratinocitos a liberar IL-8, que por el mecanismo de quimiotaxis asegura un incremento constante de la cantidad de polimorfonucleares en el surco periodontal, como produciendo estos mismos queratinocitos péptidos antimicrobianos que ayudarían a destruir patógenos periodontales ${ }^{(14)}$. Se ha podido determinar que Lactobacillus acidophilus CRL730 y Lactobacillus casei CR L431, son capaces de aumentar la capacidad fagocítica de los macrófagos ${ }^{(19)}$. Así también se ha observado la reducción significativa de la metaloproteinasa 8 (MMP-8) y altos niveles de inhibidor tisular de metaloproteinasas-1(TIMP-1) después de la administración oral de Lactobacillus reuteri (L. reuteri)(20). También se ha podido comprobar el efecto inmunomodulador del Lactobacillus brevis CD2 en la enfermedad periodontal, ya que su uso redujo los niveles de marcadores inflamatorios en saliva (metaloproteinasas, óxido nítrico, actividad sintasa, prostaglandina $E_{2}$ e interferón gama), no observando ningún efecto sobre los niveles de $\lg \mathrm{A}^{(21)}$.

\section{PROBIÓTICOS Y LA PERIODONTITIS CRÓNICA}

Mayanagui et al. evaluó si la administración oral de Lactobacillus podría alterar la población bacteriana del biofilm supra y subgingival en pacientes con periodontitis. Para lo cual un total de 66 pacientes fueron asignados aleatoriamente en dos grupos para recibir los Lactobacillus o placebo durante ocho semanas, el grupo de estudio recibió una tableta con $2,01 \times 10^{9}$ UFC/día de $L$. salivarius WB21 más xilitol en tableta y el grupo control placebo más xilitol, se recogieron muestras de biofilm dental al inicio, cuarta y octava semana. Los resultados dieron a conocer que la cantidad de las cinco bacterias periodontopatógenas seleccionados se redujeron significativamente en el biofilm subgingival a la cuarta semana. Se concluyó que la administración oral de Lactobacillus redujo la cantidad de Aggregatibacter actinomycetemcomitans (A. actinomycetemcomitans), $P$. gingivalis, Prevotella intermedia $(P$.intermedia), Treponema denticola y Tannerella forsythia, bacterias de gran relevancia en la periodontitis crónica ${ }^{(22) .}$

Vivekananda et al. en su estudio evaluó los efectos del probiótico $L$. reuteri ya sea solo o en combinación con el raspado y alisado radicular (RAR) en 30 pacientes con periodontitis crónica, el periodo de estudio fue de 42 días, realizando un estudio con diseño de boca dividida. EI RAR se realizó el día cero, a dos cuadrantes (derecho o izquierdo), mientras que los dos cuadrantes restantes se dejaron sin tratar. Las tabletas con $\left(1 \times 10^{8}\right.$ UFC DSM $17938+1 \times 10^{8}$ UFC ATCC PTA 5289$)$ de $L$. reuteri o con placebo, fueron tomados dos veces por día, desde el día 21 al día 42. Los resultados obtenidos manifiestan que el día 42 la reducción de los parámetros clínicos del índice de placa, gingival, sangrado, profundidad al sondaje y nivel de inserción clínica, así como los microbiológicos fue mayor para RAR más $L$. reuteri que para RAR más placebo y este último fue mejor que placebo solo. El estudio concluyó que hay un beneficio clínico y microbiológico por $L$. reuteri en la periodontitis crónica ${ }^{(23)}$.

Vicario et al. realizó una investigación para evaluar el efecto clínico de la administración de L. reuteri, como microorganismo probiótico en el tratamiento de la periodontitis crónica leve a moderada en 20 pacientes sistémicamente sanos, no fumadores. Los sujetos fueron asignados al azar para recibir tabletas que contienen $L$. reuteri Prodentis en concentraciones de $\left(2 \times 10^{8}\right.$ UFC) o placebo una vez al día, por 30 días. Los parámetros clínicos de índice de placa, sangrado al sondaje y profundidad al sondaje mejoraron en el grupo de estudio, mientras que en el grupo control no mostró ningún cambio estadístico significativo. El estudio concluyó que la administración oral de L. reuteri podría mejorar los parámetros clínicos periodontales en pacientes con periodontitis crónica no fumadores ${ }^{(24)}$.

Teughles et al. realizó un estudio evaluando los efectos probióticos del $L$. reuteri, en lo parámetros clínicos de profundidad y sangrado al sondaje, índice gingival y de placa y los microbiológicos de pacientes con periodontitis crónica, para lo cual utilizó una muestra de 30 pacientes, divididos en un grupo de estudio de 15 pacientes en el que se realizó el RAR y se complementó con tabletas probióticas de $L$. reuteri con $\left(1 \times 10^{8}\right.$ UFC DSM $17938+1 \times 10^{8}$ UFC ATCC PTA 5289) y un grupo control con igual número en el que se realizó RAR y se le dio un placebo. Las pastillas fueron tomadas dos veces por día durante 12 semanas. Los resultados de la investigación generaron una reducción significativa en todos los parámetros clínicos periodontal evaluados, observándose una reducción mayor de $P$. gingivalis en el grupo de estudio, así como una reducción de la bolsa periodontal y una ganancia en el nivel de inserción clínica ${ }^{(25)}$.

Tekce et al. evaluó el uso de tabletas con L.reuteri en el tratamiento adjunto de la terapia inicial de pacientes con periodontitis crónica, así como detectó el nivel de colonización de esta bacteria en las bolsas periodontales de estos pacientes. Un total de 40 pacientes fueron divididos en dos grupos de 20 pacientes, uno de grupo de estudio que recibió el procedimiento de RAR más tabletas de $L$. reuteri en la concentración de ( $2 \times 10^{8}$ UFC) dos veces por día por tres semanas, mientras que el grupo control recibió RAR más placebo. Los parámetros clínicos evaluados fueron: índice de placa, sangrado al sondaje, profundidad al sondaje y nivel de inserción clínica, así también se tomaron muestras para estudios microbiológicos al inicio y en los días 21, 90, 180 y 360 días. Los resultados del estudio determinaron mejoras significativas en todos los parámetros clínicos evaluados en el grupo de estudio comparándolo con el grupo control, en todos los tiempos evaluados, así también en el recuento de células viables y las proporciones de anaerobios obligados, con excepción del día 360 . El estudio concluyó que $L$. reuteri puede ser un coadyuvante para mejorar los resultados clínicos de la periodontitis crónica, así como para evitar una pronta recolonización microbiana ${ }^{(26)}$.

Laleman et al. evaluó el efecto coadyuvante de tres cepas probióticas, Streptococcus oralis KJ3, Streptococcus uberis KJ2 y Streptococcus ratus $\mathrm{JH} 145$, en la terapia periodontal. El grupo de 48 pacientes con periodontitis, fue dividido aleatoriamente en 24 pacientes, para el grupo de estudio en el que se realizó el RAR más la toma de tabletas con probióticos de Streptococcus que contenían aproximadamente $10^{8}$ UFC por cada bacteria, fue tomada dos veces por día durante 12 semanas, en 
el grupo control se realizó RAR más toma de placebo. Los parámetros clínicos evaluados fueron: profundidad al sondaje, sangrado al sondaje, nivel de inserción clínica, índice de placa e índice gingival, así también se realizó el estudio microbiológico. Los resultados de las mejoras en los parámetros clínicos fueron significativos en ambos grupos, aunque no hubo diferencias significativas entre ambos, a excepción del porcentaje de los sitios con placa, que fueron significativamente inferior en el grupo de estudio que el control a la evaluación de las 24 semanas. El estudio concluyó, que no se determinaron diferencias significativas al comparar el uso de un placebo o probiótico a base de Streptococcus después del $\operatorname{RAR}^{(27)}$.

Penala et al. evaluó en su ensayo controlado aleatorizado la eficacia del uso local de probióticos adjunto a RAR en el tratamiento de la periodontitis y halitosis, para lo cual en una muestra de 32 pacientes sistémicamente sanos con periodontitis crónica, fueron divididos aleatoriamente en dos grupos: grupo de estudio (RAR más probióticos) y grupo control (RAR más placebo). El probiótico usado fue L. reuteri en la concentración por cápsula de $\left(2 \times 10^{9}\right.$ UFC) y $L$. salivarius $\left(2 \times 10^{9}\right.$ UFC) el cual fue llevado localmente a la región subgingival y por enjuague de la boca con una solución de la cápsula por 15 días. Los parámetros clínicos periodontales evaluados fueron el índice de placa , índice gingival, índice de sangrado el día 0 , al mes y a los 3 meses y para la profundidad al sondaje y nivel de inserción clínica el día 0 y a los 3 meses. Los resultados obtenidos comparando los grupos determinaron una reducción de la profundidad al sondaje y ganancia del nivel de inserción clínica, con ausencia de significancia estadística pero se encontró significancia estadística para la reducción de la profundidad de bolsas moderadas para el grupo de estudio. El estudio concluyó que el uso adjunto de probióticos ofrece beneficios clínicos en la reducción de bolsas periodontales de moderada profundidad y reduce los parámetros del mal olor, aunque no existieron diferencias significativas entre los grupos ${ }^{(28)}$.

Imran et al. desarrolló un estudio clínico microbiológico, evaluando la eficacia de una bebida probiótica conteniendo Lactobacillus casei a nivel de bacterias periodontopatógenas aisladas de periodontitis. En el estudio participaron 42 personas con leve a moderada periodontitis crónica, a los cuales se le dio una bebida probiótica conteniendo Lactobacillus case (concentración: $10^{8} \mathrm{UFC} / \mathrm{mL}$ ) por un mes, tomándolo una vez por día, media hora después del desayuno. Los parámetros clínicos gingivales, niveles de placa, profundidad al sondaje, nivel de inserción clínica y sangrado al sondaje fueron registrados el día cero, al mes y a los 2 meses (este un mes después de haber dejado de usar el probiótico), se realizaron ensayos con la prueba molecular reacción en cadena de la polimerasa (PCR) multiplex, con el cual se identificó la presencia y cantidad de bacterias periodontopatógenas como $P$. gingivalis, $A$. actinomycetemcomitans y $P$. intermedia. Los resultados no encontraron significancia estadística en los parámetros clínicos, esto probablemente por la naturaleza no intervencionista del estudio, es decir no se le desarrollo RAR, solo se le indicó tomar el probiótico. Microbiológicamente se determinó que hubo una reducción significativa de los niveles de $P$. gingivalis al mes y a los dos meses y una reducción moderada de $P$. intermedia y $A$. actinomycetemcomitans. El estudio concluyó que la administración del probiótico de Lactobacillus casei redujo el número de periodontopatógenos seleccionados y que contribuiría a los efectos benéficos en las condiciones periodontales, pudiendo ser usado como coadyuvante o alternativa con la terapia periodontal, mencionando que más estudios son requeridos en este sentido o dirección ${ }^{(29)}$.

Morales et al. realizó un ensayo clínico aleatorio doble ciego, evaluando el efecto clínico del uso de probióticos en el tratamiento de la periodontitis crónica, para lo cual usó el probiótico L. rhamnosus SP1(2X 10 $10^{7}$ UFC) en polvo de disolución oral, sumado a la terapia periodontal no quirúrgica. Participan 28 pacientes sistémicamente sanos con diagnóstico de periodontitis crónica, que fueron monitorizados clínicamente desde el día 0 , a los 3 y 6 meses después de la terapia periodontal. Los parámetros periodontales evaluados eran: placa, sangrado al sondaje, profundidad al sondaje y perdida de inserción clínica. El total de participantes fueron divididos en dos grupos de 14 personas, ambos grupos recibieron terapia periodontal (RAR) y solamente el grupo de estudio recibió el probiótico, el grupo control recibió un placebo. Ambos placebo como probiótico debieron ser ingeridos una vez al día durante tres meses. Los resultados determinaron que ambos grupos mejoraron sus parámetros clínicos periodontales, pero es el grupo de estudio que redujo significativamente el porcentaje de sitios, dientes y número de participantes con profundidad al sondaje $\geq 5 \mathrm{~mm}$ entre el tiempo basal y los 6 meses postratamiento. Se concluyó que el uso de $L$. rhamnosus SP1 durante la terapia periodontal inicial genera resultados similares en los parámetros clínicos al ser comparado con el tratamiento periodontal solo(30).

Grunner et al. realizó una revisión sistemática y metanalisis sobre el manejo de probióticos en caries dental y periodontitis, analizó 50 estudios donde participaron en total 3247 personas así como los probióticos (Lactobacillus, Bifidobacterium principalmente). De sus resultados más significativos, se determinó que los probióticos reducen la profundidad de las bolsas periodontales, con diferencias de medias estandarizadas (SMD) (SMD: $-0,86 ;-1,55 /-0,17)$, así también reduce significativamente el sangrado al sondaje (SMD: $-1,15 ;-1,68 /-0,62)$ y el índice gingival (SMD: $-0,86 ;-1,52 /-0,20)$ pero no el índice de placa. El estudio concluyó en relación a la periodontitis, que los probióticos podrían ser un soporte para el manejo de la gingivitis o periodontitis, necesitando futuros estudios para determinar su eficacia y seguridad (4).

Cabezas et al. realizó una revisión sistemática y metanalisis, de la eficacia clínica de los probióticos con una terapia adjunta al tratamiento periodontal no quirúrgico de la periodontitis crónica, donde muestra resultados estadísticamente significativos en la ganancia o aumento del nivel de inserción clínica $(-0,42 \mathrm{~mm}, p=0,002)$ y reducción en el sangrado al sondaje $(-14,66, p=0,003)$ para RAR mas probiótico versus RAR solo a corto tiempo. Así también valores significativos se dan en la reducción de bolsas moderadas $(-0,18, p=0,001)$ y profundas $(-0.67, p<0,001)$. Concluyendo el uso de los probióticos como L. reuteri asociado al RAR en el tratamiento de la periodontitis crónica puede ser utilizado como complemento, aunque también manifiesta que más estudios clínicos aleatorios son necesarios para evaluar la eficacia de probióticos como terapia adjunta al RAR(31).

\section{DISCUSIÓN}

En la actualidad los probióticos vienen usándose con mucha seguridad en la medicina, para tratar afecciones intestinales y con menos continuidad en enfermedades hepáticas, pancreatitis aguda grave y para mejorar la absorción de calcio en la salud ósea, esto según Olveira ${ }^{(2)}$ pero su aplicación en las enfermedades de la cavidad bucal están siendo investigadas y en proceso de consolidación. Así es que hay estudios como la revisión de Anusha ${ }^{(1)}$, Lye ${ }^{(10)}$, que manifiestan los beneficios en la caries dental, antagonizando la acción ácida de las bacterias cariogénicas, aunque hay otros como el de Gruner(4) que manifiesta que la evidencia científica actual es insuficiente para recomendar los probióticos para tratar la caries dental. Otro problema presente en la cavidad bucal es la halitosis, estudios de revisión como el de George ${ }^{(14)}$ y Lye ${ }^{(10)}$ proponen a los probióticos como posible tratamiento contra la halitosis ya que las bacterias proteolíticas anaerobias estrictas están en relación a esta manifestación en la cavidad bucal y bacterias probióticas como la Weisella cebarei produce peróxido de hidrógeno que inhibe el crecimiento de $F$. nucleatum y bacterias productoras de compuestos volátiles sulfurados. En relación a la periodontitis las investigaciones de Gruner ${ }^{(4)}$, Gupta ${ }^{(7)}$, Lye $^{(10)}$, George $^{(14)}$, Anusha(1), Elavarasu ${ }^{(12)}$, manifiestan los beneficios de los probióticos en reducir la flora microbiana predominante en enfermedad, así como los parámetros clínicos periodontales proponiéndolos como posible producto que complementaria la terapia periodontal no quirúrgica.

Estudios acerca de los beneficios de los probióticos en la periodontitis crónica han demostrado que sí brindan un beneficio en la mejoría de los parámetros clínicos como los microbiológicos. Así Teughels ${ }^{(25)}$, en su estudio concluyó que usar probióticos como el $L$. reuteri más la terapia periodontal mejora los indicadores de salud periodontal, como reduce bolsas periodontales y gana inserción clínica en la periodontitis crónica. De la misma forma el estudio de Penala(26) usando una combinación de especies de L. reuteri y Lactobacillus salivarius para ver los efectos sumado al tratamiento convencional de la periodontitis crónica concluyó que los probióticos ofrecen un beneficio clínico en corto plazo, reduciendo la profundidad de bolsas periodontales moderadas.

En relación a que microorganismos probióticos es el que presentó mejores resultados, a la luz de la evidencia científica en producir beneficios en la periodontitis crónica, se podría mencionar a dos géneros Lactobacillus y Bifidobacterium, pero es el género Lactobacillus y la especie reuteri el que según Cabezas ${ }^{(29)}$ mejores resultados han dado en la reducción de los parámetros clínicos de la periodontitis crónica. Según Talarico ${ }^{(32)}$ Lactobacillus reuteri secreta dos bacteriocinas: reuterina y reuterociclina, que inhiben el crecimiento de una ancha variedad de patógenos. Así también tiene una fuerte capacidad de adherirse a la célula huésped, compitiendo con bacterias patógenas, así como presentan una acción antiinflamatoria inhibiendo citoquinas proinflamatorias. Los resultados positivos de $L$. reuteri no se observan con otras especies de Lactobacillus estudiados.

\section{CONCLUSIONES}

Por lo expuesto y ante la falta de suficiente evidencia científica, que sustente el uso de los probióticos en las enfermedades periodontales, todavía no se podría concluir sobre sus beneficios a nivel periodontal, 
pero los resultados de varios estudios estarían evidenciando efectos favorables en la terapia periodontal como coadyuvante, siendo el $L$. reuteri la cepa probiótica con un posible mayor beneficio en el tratamiento de la periodontitis crónica.

\section{FUENTE DE FINANCIAMIENTO}

Autofinanciado

\section{CONFLICTO DE INTERÉS}

Los autores manifiestan no tener ningún conflicto de interés personal u organizacional que pudiera influir en la revisión.

\section{Bibliografía}

1. Anusha RL, Umar D, Basheer B, Baroudi K. The magic bugs in oral cavity: Probiotics. J Adv Pharm Technol Res. 2015;6:43-47.

2. Olveira G, Gonzales-Molero I. Probióticos y prebióticos en la práctica clínica. Nutr Hosp. 2007;22(supl. 2):26-34.

3. Zalba JI, Flichy-Fernandez AJ. Empleo de probióticos en odontología. Nutr Hosp. 2013;28(supl. 1):49-50.

4. Gruner D, Paris S, Schwendicke F. Probiotics for managing caries and periodontitis: Systematic review and meta-analysis. J Dent. 2016;48:16-25. 5. Amores R, Calvo A. Probióticos. Rev Esp Quimioterap. 2004;17(2):131-139. 6. Sanders ME. Probiotics: considerations for human health. Nutr Rev. 2003;61(3):91-99. 7. Gupta, G. Probiotics and periodontal health. J Med Life. 2011;4(4):387-394 8. Devine D, Marsh P. Prospects for the development of probiotics and prebiotics for oral applications. J Oral Microbiol. 2009;1:10

9. Jepsen K, Jepsen S. Antibiotics/antimicrobials: systemic and local administration in the therapy of mild to moderately advanced periodontitis. Periodontol 2000. 2016;71:82-112

10. Shi LH, Balakrishnan K, Thiagarajah K, Mohd Ismail NI, Yin OS. Beneficial properties of probiotics. Trop Life Sci Res. 2016;27(2):73-90

11. Allaker RP, lan-Douglas CW. Non conventional therapeutics for oral infections. Virulence. 2015;6(3):196-207.

12. Elavarasu S, Suthanthiran T, Thangavelu A, Kanagaraj SS, Mohandas L, Sekar $\mathrm{S}$. Evaluation of efficacy of probiotic (Bifilac) on Porphyromonas gingivalis. In vitro study. J Pharm Bioall Sci. 2016;8(Suppl S1):45-47.

13. Ramos PD, Moromi NH, Martinez CE. Porphyromonas gingivalis: patógeno predominante en la periodontitis crónica. Odontol Sanmarquina. 2011:14(1):34-38.

14. George VT, Varghese MM, Vaseem MS, Thomas A, Ittycheria PG, Sreejith CK. The promising future of probiotics : A new era in periodontal therapy. J Int Oral Health. 2016;8(3):404-408.

15. Lopez M, Domingo D. Antibioticoterapia con probióticos. Rev Esp Quimioterap. 2007; 20(2):170-181.

16. Pangsomboon K, Kaewnopparat S Pitakpornpreecha T, Srichana T. Antibacterial activity of a bacteriocin from Lactobacillus paracasei HL32 against Porphyromonas gingivalis. Arch Oral Biol. 2006; 51(9):784-793.

17. Khalaf, H, Nakka, S, Sandén, C. Antibacterial effects of lactobacillus and bacteriocin NC8 $\alpha \beta$ on the periodontal pathogen Porphyromonas gingivalis. BMC Microbiology. 2016;16(188):1-11.

18. Stamatova I, Meurman J. Probiotics: Health benefits in the mouth. Am J Dent. 2009; 22(6):329-338

19. Iniesta M, Montero E, Zurbriggen M, Herrera D. El control del biofilm y los probióticos. Rev Soc Esp Periodon Osteoint. 2012;22(4):273-280.

20. Ince, G, Gürsoy, Ipci SD, Cakar G, Emekli-Alturfan E, Yilmaz S. Clinical and biochemical evaluation of lozenges containing Lactobacillus reuteri as an adjunct to non-surgical periodontal therapy in chronic periodontitis. J Periodontol. 2015;86(6):746-754
21. Riccia DN, Bizzini F, Perilli MG, Polimeni A, Trinchieri V, Amicosante G, et al. Anti-inflammatory effects of Lactobacillus brevis (CD2) on periodontal disease. Ora Dis 2007:13(4):376-385.

22. Mayanagui G, Kimura M, Nakaya S, Hirata H, Sakamoto M, Benno Y, et al Probiotic effects of orally administered Lactobacillus salivarius WB21 containing tablets on periodontopathic bacteria: a double-blinded, placebo-controlled, randomized clinical trial. J Clin Periodontol. 2009;36:506-513.

23. Vivekananda M, Vandana K, Bhat KG. Effect of the probiotic Lactobacillus reuteri (Prodentis) in the management of periodontal disease: a preliminary randomized clinical trial. J Oral Microbiol. 2010;2:1-9

24. Vicario M, Santos A, Violant D, Nart T, Giner L. Clinical changes in periodontal subjects with the probiotic Lactobacillus reuteri Prodentis: a preliminary randomized clinical trial. Acta Odontol Scand. 2013;71(4): 813-819.

25. Teughels W, Durukan A, Ozcelik O, Pauwels M, Quirynen M, Cenk Haytac MC. Clinical and microbiological effects of Lactobacillus reuteri probiotics in the treatment of chronic periodontitis a: randomized placebo-controlled study. J Clin Periodontol. 2013:40:1025-1035.

26. Tekce M, Ince G, Gursoy H, Dirikan L, Cakar G, Kadir T, et al. Clinical and microbiological affects of probiotic lozenges in the treatment of chronic periodontitis : a 1 years follow-up study. J Clin Periodontol. 2015;42(4):363-372.

27. Laleman L, Yilmaz E, Ozcelik O, Haytac C, Pauwels M, Herrero E, et al. The effect of a streptococci containing probiotic in periodontal therapy: a randomized controlled trial. J Clin Periodontol. 2015;42(11):1032-1041.

28. Penala S, Kalakonda B, Pathakota KR, Jayakumar A, Koppolu P, Lakshmi BV, et al. Efficacy of local use of probiotics as an adjunct to scaling and root planning in chronic periodontitis and halitosis: Arandomized controlled trial. J Res Pharm Pract. 2016;5:86-93.

29. Imran F, Das S, Padmanabhan S, Rao R, Suresh A, Bharath D. Evaluation of the efficacy of probiotics drink containing Lactobacillus casei on the levels of periodontopathic bacteria in periodontitis: A clinic-microbiologic study. Indian J Dent Res. 2015;26:462-468

30. Morales A, Galaz C, González J, Silva N, Hernandez M, Godoy C, et al. Efecto clínico del uso de probiótico en el tratamiento de la periodontitis crónica: ensayo clínico. Rev Clin Periodoncia Implantol Rehabil Oral. 2016;9(2):146-152.

31. Martin-Cabezas R, Davideau JL, Tenenbaum H, Huck O. Clinical efficacy of probiotics as an adjuntive therapy to non-surgical periodontal treatment of chronic periodontitis a sytematic review and meta-analysis. J Clin Periodontol. 2016;43(6):520-530.

32. Talarico TL, Casas IA, Chung TC, Dobrogosz WJ. Production and isolation of reuterina a growth inhibitor produced by Lactobacillus reuteri. Antimicrob Agents Chemother. 1988;32(12):1854-1858. 